\title{
Numerical simulations of the internal shock model in magnetized relativistic jets of blazars
}

\author{
Jesus Rueda-Becerril* and Petar Mimica and Miguel A. Aloy \\ Departamento de Astronomía y Astrofísica, Universidad de Valencia, E-46100 Burjassot \\ (Valencia), Spain \\ E-mail: jesus.rueda@uv.es, petar.mimica@uv.es, miguel.a.aloy@uv.es
}

\begin{abstract}
The internal shocks scenario in relativistic jets is used to explain the variability of the blazar emission. Recent studies have shown that the magnetic field significantly alters the shell collision dynamics, producing a variety of spectral energy distributions and light-curves patterns. However, the role played by magnetization in such emission processes is still not entirely understood. In this work we numerically solve the magnetohydodynamic evolution of the magnetized shells collision, and determine the influence of the magnetization on the observed radiation. Our procedure consists in systematically varying the shell Lorentz factor, relative velocity, and viewing angle. The calculations needed to produce the whole broadband spectral energy distributions and light-curves are computationally expensive, and are achieved using a high-performance parallel code.
\end{abstract}

Swift: 10 Years of Discovery

2-5 December 2014

La Sapienza University, Rome, Italy

${ }^{*}$ Speaker. 


\section{Introduction}

Blazars are a class of radio-loud active galactic nuclei (AGNs) whose jets are pointing near the line of sight to the observer [1], and are known for showing the most rapid variability of all AGNs. Extensive observations indicate that the jets of blazars are relativistic. Their most remarkable characteristic is the presence of flares in the X-and $\gamma$-ray bands, usually wih a duration of the order of a few hours. The internal shock (IS) model [2], invoked to explain this variability [3, 4, 5], is an idealized model where an intermittently working central engine ejects shells of magnetized plasma which collide due to their velocity differences. As a consequence internal shocks are formed, accelerating particles at the shock fronts and producing the non-thermal, highly variable radiation.

Our long-term objective is the study of the influence of the magnetic field on the observed emission using numerical simulations. In [6] we studied a large number of shell collisions with different magnetization levels. In [7] we focused on a limited number of parameters such as the observer viewing angle, $\theta$, the bulk Lorentz factor of the slower shell, $\Gamma_{R}$, and the relative Lorentz factor, $\Delta g:=\left(\Gamma_{L} / \Gamma_{R}\right)-1$, where $\Gamma_{L}$ is the bulk Lorentz factor of the faster shell. In the present work we summarize some of the results studied therein.

We describe the common numerical setup in Sec. 2. The results are shown in Sec. 3 and discussed in Sec. 4.

\section{Numerical Setup}

A modified version of the code SPEV [8] was employed to calculate the non-thermal emission from ISs. We assume a cylindrical shell geometry and perform all the calculations in the rest frame of the shocked fluid (RFSF). The shell interaction was simplified as a one-dimensional Riemann problem focussing our resources on a more detailed treatment of the non-thermal radiation. Our method consists of three steps that we sum up in the following paragraphs.

Solution of the Riemann problem. Employing an exact relativistic magnetohydrodynamics (RMHD) Riemann solver we determine the properties of the internal shock waves. We follow the procedure described in [6] to set-up the shells and to extract the information needed for the steps 2 and 3.

Non-thermal particles transport and evolution. The particles are injected behind the shock fronts following the prescription of $[9,10,6]$. We assume that a fraction of the thermal electrons are accelerated into a power-law distribution at high energies, and that their energy density is a fraction of the internal energy density of the shocked fluid. In the RFSF the shocks are propagating away from the initial discontinuity, injecting and leaving non-thermal particles behind. We evolve the energy distribution of non-thermal electrons taking into account synchrotron and inverse-Compton (IC) losses. See [6] for more details.

Radiative transfer. The total emissivity at each point is assumed to be a combination of the following emission processes: (1) synchrotron radiation, (2) IC upscattering of an internal (SSC) and an external radiation field (EIC).

\section{Results}

We group our models according to the initial shell magnetization, $\sigma:=B^{2} / 4 \pi \rho \Gamma^{2} c^{2}$. Hereafter 
the subscripts $L$ and $R$ will denote left (faster) and right (slower) shells, respectively. We compute the spectra for a typical source located at $z=0.5$. In the rest of this section we will present some of the final SEDs resulting from our simulations. A larger collection is shown in [7]. The SED of each model has been averaged over the time interval $0-10^{6} \mathrm{~s}$.

\subsection{Weakly-magnetized models}

The SEDs computed for the models with $\sigma_{L}=\sigma_{R}=10^{-6}, \Gamma_{R}=10, \theta=5^{\circ}$ and varying $\Delta g$, are shown in the upper left panel of Fig. 1. The spectra show that with increasing $\Delta g$ the IC component also increases, up to three orders of magnitude. In order to see the effects on each emission process, the synchrotron, SSC and EC components for $\Delta g=0.5,2.0$ are shown as dashed, dot-dashed and dot-dot-dashed lines, respectively. While the three spectral components (synchrotron, SSC and EC) have approximately the same order of magnitude for $\Delta g=0.5$, for $\Delta g=2.0$ the SSC is almost two orders of magnitude more luminous than the other two.

\subsection{Moderately-magnetized models}

The SEDs of the family of models $\sigma_{L}=\sigma_{R}=10^{-2}, \Delta g=1.0$ and $\theta=5^{\circ}$ are presented in the upper right panel of Fig. 1. As in the previous section, synchrotron, SSC and EC components are shown as dashed, dot-dashed and dot-dot-dashed lines, respectively, but now for the models with $\Gamma_{R}=10,17,25$. The synchrotron component for $\Gamma_{R}=10$ is $\simeq 20$ times brighter than the SSC one, in contrast to the EC which is 100 times dimmer. For $\Gamma_{R}=25$ the EC is of the same order of magnitude of SSC and synchrotron. The latter two decrease one order of magnitude between the models with $\Gamma_{R}=10$ and $\Gamma_{L}$, while the EC grows by almost one order of magnitude. This follows from the fact that the number of electrons and the comoving magnetic field strength decrease with the increasing $\Gamma_{R}[6]$, which means that there are less synchrotron photons and less electrons which can scatter them in the SSC process. We model the Klein-Nishina cutoff as a sharp cutoff, and its effect can be seen at $\simeq 10^{23} \mathrm{~Hz}$, where all the EC spectra coincide. From the inset we can see that there is no significant change in the flux of $\gamma$-photons, although the spectral index softens for increasing $\Gamma_{R}$.

\subsection{Strongly-magnetized models}

The third family consists of the strongly magnetized models where $\sigma_{L}=1$ and $\sigma_{R}=0.1$. The corresponding SEDs of this series of models (lower panel of Fig. 1 are analogous to the models of Sec. 3.2.As we can see, for $\Gamma_{R}=10$ the synchrotron component is $\simeq 100$ times brighter than the IC. For $\Gamma_{R}=25$ this difference decreases to one order of magnitude. Once again, EIC component rises with rising $\Gamma_{R}$, to the point in which it begins to be comparable to the synchrotron component, developing an IC hump. The EIC spectra intersect in a single point due to our treatment of the Klein-Nishina cutoff. In the inset we can see that the flux of $\gamma$-ray photons does not change appreciably in this family of models. Table 1 lists a number of physical parameters in the reverse shock (RS) of the present models. We see that the electrons in the RS of the strongly magnetized models are fast-cooling. In fact, for $\Delta g=1.5$ the injected electron spectrum is almost mono-energetic. 

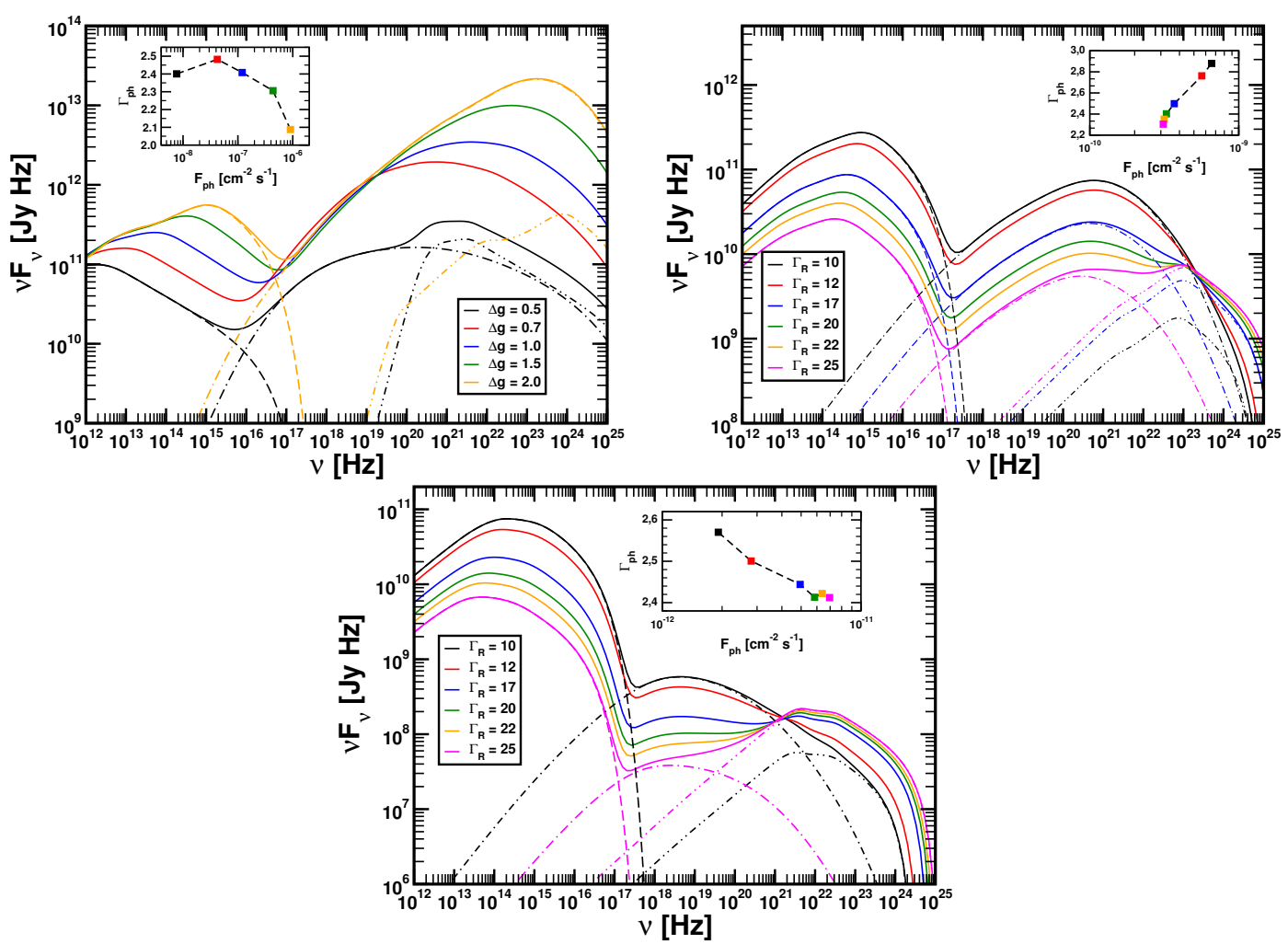

Figure 1: Upper left panel: Averaged spectra resulting from collisions of weakly magnetized shells $\left(\sigma_{L}=\sigma_{R}=10^{-6}\right)$. Upper right panel: Same as the upper left panel, but for moderately magnetized shells $\left(\sigma_{L}=\sigma_{R}=10^{-2}\right)$ and varying $\Gamma_{R}$. Lower panel: Same as upper right panel, but for strongly magnetized shells $\left(\sigma_{L}=1, \sigma_{R}=0.1\right)$. The inset in each panel shows the photon spectral slope $\Gamma_{p h}$ as a function of the photon flux $F_{p h}$ in the $\gamma$-ray band (see Sec. 3.2). Colors of the points correspond to the line colors in the main plot. For selected models, the synchrotron, SSC and EIC contributions (dashed, dot-dashed and dot-dot-dashed lines, respectively) are shown.

\begin{tabular}{rrrrccccc}
\hline \hline$\Delta g$ & $\Gamma$ & $r_{r}$ & $\frac{B_{r}}{1 \mathrm{G}}$ & $\frac{Q_{r, 11}}{\mathrm{~cm}^{-3} \mathrm{~s}^{-1}}$ & $\frac{\gamma_{1 r}}{10^{2}}$ & $\frac{\gamma_{2 r}}{10^{4}}$ & $\frac{t_{c r r}^{\prime}}{10^{3} \mathrm{~s}}$ & $\frac{\gamma_{c r}}{\gamma_{1 r}}$ \\
\hline 0.5 & 12.7 & 1.26 & 53.51 & 0.11 & 0.66 & 0.64 & 34.6 & 0.12 \\
0.7 & 12.8 & 1.46 & 54.72 & 1.03 & 2.29 & 0.63 & 34.1 & 0.03 \\
1.0 & 13.0 & 1.75 & 55.84 & 7.33 & 7.25 & 0.62 & 33.6 & 0.01 \\
1.5 & 13.2 & 2.22 & 56.63 & 68.00 & 26.38 & 0.62 & 32.9 & 0.003 \\
2.0 & 13.3 & 2.67 & 56.82 & 112900.75 & 61.68 & 0.62 & 32.5 & 0.001 \\
\hline
\end{tabular}

Table 1: Physical parameters in the RS shocked region for the family of strongly magnetized shells with different $\Delta g$. The bulk Lorentz factor of both shocked regions is denoted by $\Gamma$, while $r, B, Q, \gamma_{1}$ and $\gamma_{2}$ denote its compression ratio, comoving magnetic field, comoving number of electrons injected per unit volume and unit time, and lower and upper cutoffs of the injected electrons in the RS (see Eq. 11 of MA12). $Q_{11}=Q \times 10^{-11} \cdot t_{c r}^{\prime}:=\Delta r^{\prime} /\left(c\left|\beta^{\prime}\right|\right)$ is the shock crossing time, where $\Delta r^{\prime}$ and $\beta^{\prime}$ are the shell width and the shock velocity in the frame moving with the contact discontinuity separating both shocks (section 2 of MA12). $\gamma_{c}:=\gamma_{2} /\left(1+v_{0} \gamma_{2} t_{c r}^{\prime}\right)$ is the cooling Lorentz factor of an electron after a dynamical time scale (shock crossing time). $v_{0}:=(4 / 3) c \sigma_{T}\left(u_{B}^{\prime}+u_{\text {ext }}^{\prime}\right) /\left(m_{e} c^{2}\right)$ is the cooling term, where $\sigma_{T}$ is the Thomson cross section and the primed quantities are measured in the comoving frame. When $\gamma_{c} / \gamma_{1} \gg(\ll) 1$ the electrons are slow (fast) cooling. Note that the $Q_{11}$ for $\Delta g=2.0$ is much larger than $Q_{11}$ of the other models because $\gamma_{1} \simeq \gamma_{2}$. 

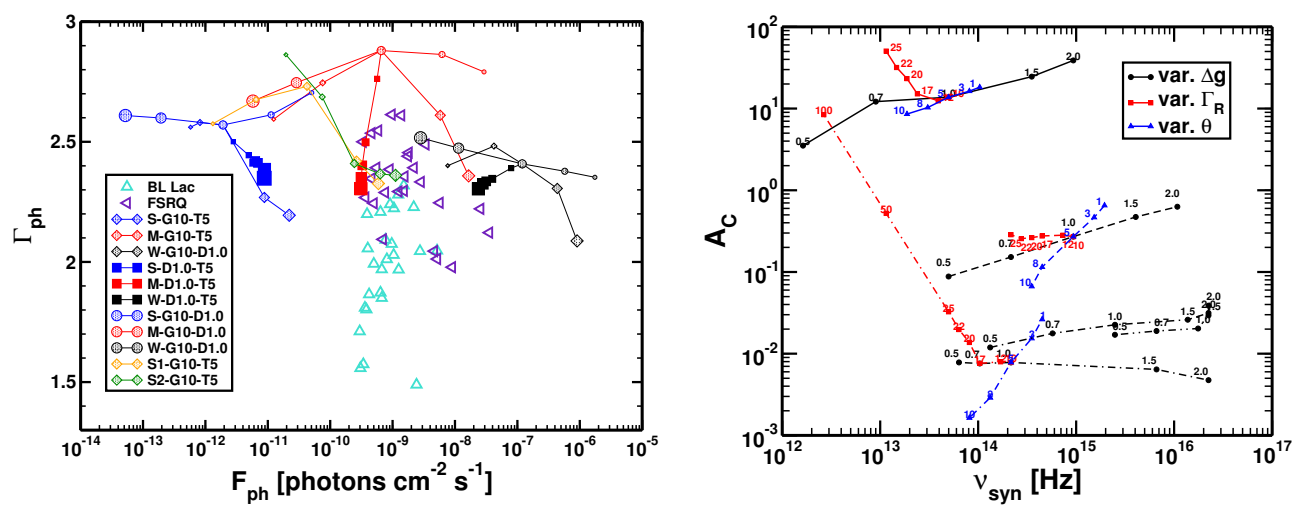

Figure 2: Left panel: Spectral slope $\Gamma_{p h}$ for the photon energies $>200 \mathrm{MeV}$ as a function of the photon flux for energies $>100 \mathrm{MeV}$ [11]. The symbols joined by lines represent our numerical models, while cyan and magenta triangles represent BL Lacs and FSRQs at redshift $z \simeq 0.5$ from 2LAC [12]. Right panel: Compton dominance $A_{C}$ as a function of the synchrotron peak frequency $v_{\mathrm{syn}}$ for all the models studied in [7].

\section{$3.4 \gamma$-rays spectral slope}

A linear least-squares algorithm is used to deduce the $\gamma$-ray spectral slope $\Gamma_{p h}$. Due to the fact that we are not modeling the Klein-Nishina part of the spectrum, we only performed the calculations of $\Gamma_{p h}$ for those models that do not show a large drop-off in the photon flux. In Fig. 2 we show $\Gamma_{p h}$ as a function of the photon flux for energies $>0.2 \mathrm{GeV}$, where $F_{p h}$ is the photon flux for photon energies $>0.1 \mathrm{GeV}$ [11]. We compare our results with sources found in 2LAC [12], restricting the comparsion to sources with $0.4 \leq z \leq 0.6$. In Fig. 2 we see that weakly and moderately magnetized models overlap with the observations, with more weakly than moderately magnetized models falling within the observed part of the parameter space.

\subsection{Compton dominance $A_{C}$}

Another way to correlate magnetization with observed properties can be found by representing the Compton dominance $A_{C}$ as a function of the ratio of IC-to-synchrotron peak frequencies $v_{I C} / v_{s y n}$. Models with intermediate or low magnetization occupy a range of $A_{C}$ roughly compatible with observations [13], while the strongly magnetized models tend to have values of $A_{C}$ that are probably incompatible with those observed in actual sources, unless collisions in blazars happen at much larger Lorentz factors than currently thought.

\section{Conclusions}

We have shown that the SEDs of FSRQs and BL Lacs strongly depend on the magnetization of the emitting plasma. Our models predict a more complex phenomenology than what is currently supported by the observational data. A conservative conclusion is that the observations of actual sources restrict the magnetization of their colliding shells to, at most, moderate values (i.e., $\left.\sigma \lesssim 10^{-1}\right)$. However, if the actual shells are strongly magnetized, than the observations restrict the difference in colliding shell magnetization to be less than a factor $\sim 10$. We find that FSRQs have observational properties corresponding to models with negligible or moderate magnetic fields. BL 
Lacs with moderate peak synchrotron frequencies $v_{s y n} \lesssim 10^{16} \mathrm{~Hz}$ and Compton dominance parameter $0.1 \gtrsim A_{C} \gtrsim 1$ display properties that can be reproduced with models with moderate and uniform magnetization $\left(\sigma_{L}=\sigma_{R}=10^{-2}\right)$. We find that a fair fraction of the blazar sequence can be explained in terms of the intrinsically different magnetization of the colliding shells. We observe that the change in the photon spectral index $\left(\Gamma_{\mathrm{ph}}\right)$ in the $\gamma$-ray band can be a powerful observational proxy for the actual values of the magnetization and of the relative Lorentz factor of the colliding shells. Values $\Gamma_{\mathrm{ph}} \gtrsim 2.6$ result in models where the flow magnetization is $\sigma \sim 10^{-2}$, whereas strongly magnetized shell collisions $(\sigma>0.1)$ as well as weakly magnetized models may yield $\Gamma_{\mathrm{ph}} \lesssim 2.6$.

\section{Acknowledgments}

We acknowledge the support from the European Research Council (grant CAMAP-259276), and the partial support of grants AYA2013-40979-P, CSD2007-00050 and PROMETEO-2009-103, and to the Consejo Nacional de Ciencia y Tecnología (MEXICO) for a doctoral fellowship to J.M.R.B.

\section{References}

[1] C.M. Urry \& P. Padovani, Unified Schemes for Radio-Loud Active Galactic Nuclei, PASP 107 (803) 1995.

[2] M.J. Rees \& P. Meszaros, Unsteady outflow models for cosmological gamma-ray bursts, ApJL 430 (L93) 1994.

[3] P. Mimica et al., Synthetic X-ray light curves of BL Lacs from relativistic hydrodynamic simulations, AEA 418 (947) 2004.

[4] P. Mimica et al., Which physical parameters can be inferred from the emission variability of relativistic jets?, AEA 441 (103) 2005.

[5] M. Spada et al., Internal shocks in the jets of radio-loud quasars, MNRAS 325 (1559) 2001.

[6] P. Mimica \& M.A. Aloy, Radiative signature of magnetic fields in internal shocks, MNRAS $\mathbf{4 2 1}$ (2635) 2012.

[7] J.M. Rueda-Becerril, P. Mimica \& M.A. Aloy, The influence of the magnetic field on the spectral properties of blazars, MNRS 438 (1856) 2014.

[8] P. Mimica et al., Spectral Evolution of Superluminal Components in Parsec-Scale Jets, ApJ 696 (1142) 2009.

[9] M. Böttcher \& C.D. Dermer, Timing Signatures of the Internal-shock Model for Blazars, ApJ 711 (445) 2010.

[10] M. Joshi \& M. Böttcher, Time-dependent Radiation Transfer in the Internal Shock Model Scenario for Blazar Jets, ApJ 727 (21) 2011.

[11] A.A. Abdo et al., Bright Active Galactic Nuclei Source List from the First Three Months of the Fermi Large Area Telescope All-Sky Survey, ApJ 700 (597) 2009.

[12] M. Ackermann et al., The Second Catalog of Active Galactic Nuclei Detected by the Fermi Large Area Telescope, ApJ $\mathbf{7 4 3}$ (171) 2011.

[13] J.D. Finke, Compton Dominance and the Blazar Sequence, ApJ 763 (134) 2013. 